\title{
Reeb dynamics detects odd balls
}

\author{
HANSJÖRG GEIGES AND KAI ZEHMISCH
}

\begin{abstract}
We give a dynamical characterisation of odd-dimensional balls within the class of all contact manifolds whose boundary is a standard even-dimensional sphere. The characterisation is in terms of the non-existence of short periodic Reeb orbits.
\end{abstract}

Mathematics Subject Classification (2010): 53D35 (primary); 37C27, 37J55, 57R17 (secondary).

\section{Introduction}

\subsection{Definitions and the main result}

Let $(M, \alpha)$ be a compact, connected contact manifold (with a fixed choice of contact form $\alpha$ ) of dimension $2 n+1, n \in \mathbb{N}$, whose boundary $\partial M$ is diffeomorphic to $S^{2 n}$.

We write $\inf _{0}(\alpha)$ for the infimum of all positive periods of contractible closed orbits of the Reeb vector field $R_{\alpha}$. When there are no closed contractible Reeb orbits, we have $\inf _{0}(\alpha)=\infty$, otherwise $\inf _{0}(\alpha)$ is a minimum and in particular positive.

Our main result will be a criterion for $M$ to be diffeomorphic to a ball in terms of $\inf _{0}(\alpha)$ and an embeddability condition on $\partial M$. To formulate this condition, we introduce the following terminology.

Definition 1.1. (a) Write $D$ for the closed unit disc in $\mathbb{R}^{2}$. The $(2 n+1)$-dimensional manifold (with boundary)

$$
Z:=\mathbb{R} \times D \times \mathbb{C}^{n-1}
$$

with contact form

$$
\alpha_{\text {cyl }}:=\mathrm{d} b+\frac{1}{2}\left(x_{0} \mathrm{~d} y_{0}-y_{0} \mathrm{~d} x_{0}\right)-\sum_{j=1}^{n-1} y_{j} \mathrm{~d} x_{j}
$$

H. G. and K. Z. are partially supported by DFG grants GE 1245/2-1 and ZE 992/1-1, respectively. Received April 10, 2014; accepted in revised form July 16, 2014.

Published online February 2016. 
(with the obvious denomination of cartesian coordinates) will be referred to as the contact cylinder.

(b) We say that $\partial M$ admits a contact embedding into the contact cylinder $Z$ if there is an embedding $\varphi$ of a collar neighbourhood of $\partial M \subset M$ into $\operatorname{Int}(Z)$ with $\varphi^{*} \alpha_{\text {cyl }}=\alpha$ and with the image of the collar under $\varphi$ contained in the interior of $\varphi(\partial M)$, i.e. in the compact component of $Z \backslash \varphi(\partial M)$.

Theorem 1.2. Assume that the boundary $\partial M \cong S^{2 n}$ of a contact manifold $(M, \alpha)$ as above admits a contact embedding into the contact cylinder, and $\inf _{0}(\alpha) \geq \pi$. Then $M$ is diffeomorphic to a ball.

This theorem has been proved for $\operatorname{dim} M=3$ by Eliashberg and Hofer [5]. In that paper, they also announced the theorem for the higher-dimensional case, but a proof has never been published. They formulated the higher-dimensional case under the additional homological assumption $H_{2}(M ; \mathbb{R})=0$; this condition, as we shall see, is superfluous.

For simplicity, we shall assume throughout that $n \geq 2$, although a large part of our argument also works for $n=1$. Our proof shows that $(M, \alpha)$ is diffeomorphic to a ball whenever $\partial M \cong S^{2 n}$ admits a contact embedding into the cylinder $Z_{r}:=$ $\mathbb{R} \times D_{r}^{2} \times \mathbb{C}^{n-1}$ of radius $r$, and $\inf _{0}(\alpha)>\pi r^{2}$. Given a contact embedding into $Z=$ $Z_{1}$, it may be regarded as an embedding into a cylinder of slightly smaller radius. Hence, even though the proof below will be based on the assumption $\inf _{0}(\alpha)>\pi$, the result holds under the weaker assumption $\inf _{0}(\alpha) \geq \pi$.

\subsection{Idea of the proof}

The contact embedding $\varphi$ of $\partial M$ into the contact cylinder $Z$ allows us to form a new contact manifold $\widehat{\mathbb{R}}^{2 n+1}$ by removing the bounded component of $\mathbb{R}^{2 n+1} \backslash \varphi(\partial M)$ and gluing in $M$ instead. Similarly, we write $\widehat{Z}$ for the cylinder $Z$ with $M$ glued in. We shall be studying the moduli space $\mathcal{W}$ of holomorphic discs $u=(a, f): \mathbb{D} \rightarrow$ $\mathbb{R} \times \widehat{\mathbb{R}}^{2 n+1}=: W$ in the symplectisation $W$ of $\widehat{\mathbb{R}}^{2 n+1}$, where the discs are subject to certain boundary and homological conditions. (We always write $\mathbb{D}$ for the closed unit disc in $\mathbb{C}$ when regarded as the domain of definition of our holomorphic discs.) It will turn out that $f(\mathbb{D})$ is always contained in $\widehat{Z}$. We then have the following dichotomy. Either the evaluation map

$$
\begin{aligned}
& \mathrm{ev}: \mathcal{W} \times \mathbb{D} \longrightarrow \widehat{Z} \\
& ((a, f), z) \longmapsto f(z)
\end{aligned}
$$

is proper and surjective, i.e. gives a filling, in which case topological arguments involving the $h$-cobordism theorem can be used to show that $M$ must be a ball. Otherwise there will be breaking of holomorphic discs, which entails the existence of short contractible periodic Reeb orbits as in Hofer's paper [12]. 


\subsection{Remarks}

(1) The bound $\pi$ in Theorem 1.2 is optimal. Inside $Z$ one can form the connected sum as described by Weinstein [21], $c f$. [8, Section 6.2], with any contact manifold, producing a belt sphere of radius $r_{0}$ smaller than, but arbitrarily close to 1 . Inside this belt sphere one finds a periodic orbit of length $\pi r_{0}^{2}$.

(2) In the 3-dimensional case, Theorem 1.2 can be strengthened. If $\inf _{0}(\alpha) \geq \pi$, then there are in fact no closed Reeb orbits at all. (This was part of the formulation of the theorem in [5].) In this 3-dimensional case, the holomorphic discs project to embedded discs in $\widehat{Z}$, where they produce a foliation by discs transverse to the Reeb direction, see [5, Section 2]. This precludes closed orbits.

(3) The existence of a foliation by discs as in (2) implies that there cannot even be trapped Reeb orbits, i.e. orbits that are bounded in forward or backward time. In [9] we show in joint work with Nena Röttgen that this is a purely 3-dimensional phenomenon. In higher dimensions it is possible to have a Reeb dynamics on Euclidean space, standard outside a compact set, with trapped orbits but no periodic ones.

(4) One may consider manifolds $M$ with disconnected boundary (and boundary components different from $S^{2 n}$ ). The requirement of a contact embedding into the contact cylinder $Z$ is made for each component of $\partial M$ individually. By translating the images of these components in the $\mathbb{R}$-direction one may then assume without loss of generality that they are not nested. The collection $\varphi(\partial M)$ of these images is contained in a large ellipsoid $E$ inside $\operatorname{Int}(Z)$. The manifold obtained from $E$ by removing the interiors of the components of $\varphi(\partial M)$ and gluing in $M$ instead has non-trivial fundamental group: by taking a path in $M$ joining two boundary components, and a second path joining these two boundary points in the exterior of $\varphi(\partial M) \subset E$, one creates an essential loop. It follows that this manifold contains a contractible Reeb orbit of period smaller than $\pi$. This orbit must in fact be contained entirely in $M$, since the Reeb flow on $Z$ is positively transverse to any hypersurface $\{b\} \times D^{2 n}$.

In other words, Theorem 1.2 provides a means of detecting contractible periodic orbits on non-compact manifolds or manifolds with boundary. See $[2,3,20]$ for related work.

ACKnowledgements. We thank Peter Albers for useful conversations about compactness questions, and Chris Wendl for drawing our attention to Frédéric Bourgeois's work on transversality in the setting of symplectisations. A part of the work on this paper was done during the workshop on Legendrian submanifolds, holomorphic curves and generating families at the Académie Royale de Belgique, August 2013, organised by Frédéric Bourgeois. 


\section{Symplectisations of contactisations}

The contact cylinder $Z$ may be regarded as the contactisation of the exact symplectic manifold $D \times \mathbb{C}^{n-1} \subset \mathbb{C}^{n}$. In the latter, we have the obvious holomorphic discs $D \times\{*\}$. In order to lift these to holomorphic discs in the symplectisation of $Z$, it is advantageous to proceed in two steps: first lift them to holomorphic discs in $\mathbb{C} \times D \times \mathbb{C}^{n-1}$, and then transform them to holomorphic discs in the symplectisation $\mathbb{R} \times Z$ using an explicit biholomorphism

$$
\Phi: \mathbb{R} \times \mathbb{R} \times D \times \mathbb{C}^{n-1} \longrightarrow \mathbb{C} \times D \times \mathbb{C}^{n-1} .
$$

The desired boundary condition for the holomorphic discs on the left-hand side gives us the boundary conditions for the holomorphic discs on the right.

This allows one to transform a Cauchy-Riemann problem on the left with respect to a 'twisted' almost complex structure (which preserves the contact hyperplanes and pairs the Reeb with the symplectisation direction) into a Poisson problem on a single real-valued function.

This idea is implicit in [5, page 1320] and has also been used in [19, Proposition 5]. Before we turn to our specific situation, we discuss this transformation in slightly greater generality.

\subsection{Lifting holomorphic discs}

Let $\left(V, J_{V}\right)$ be a Stein manifold of complex dimension $n$. We write $\psi$ for a plurisubharmonic potential on $V$, so that $\omega_{V}:=-\mathrm{d}\left(\mathrm{d} \psi \circ J_{V}\right)$ is a Kähler form on $V$. In fact, what is really relevant for the following discussion is the existence of such a potential, not the integrability of $J_{V}, c f$. [10, Section 3.1]. Write $\lambda:=-\mathrm{d} \psi \circ J_{V}$ for the primitive 1-form of the symplectic form $\omega_{V}$.

The contactisation of $V$ is $(\mathbb{R} \times V, \alpha:=\mathrm{d} b+\lambda)$, where $b$ denotes the $\mathbb{R}$ coordinate. Notice that $\partial_{b}$ is the Reeb vector field of the contact form $\alpha$. A symplectisation of this manifold is

$$
(\mathbb{R} \times \mathbb{R} \times V, \omega:=\mathrm{d}(\tau \alpha)),
$$

where $\tau$ is a strictly increasing smooth positive function on the first $\mathbb{R}$-factor (whose coordinate we shall denote by $a$ ). A compatible almost complex structure $J$ on this symplectic manifold, which in addition preserves the contact hyperplanes

$$
\operatorname{ker} \alpha=\left\{v-\lambda(v) \partial_{b}: v \in T V\right\}
$$

on $\{a\} \times \mathbb{R} \times V$, is given by

$$
J\left(\partial_{a}\right)=\partial_{b} \quad \text { and } \quad J\left(v-\lambda(v) \partial_{b}\right)=J_{V} v-\lambda\left(J_{V} v\right) \partial_{b} .
$$

If $J_{V}$ is not integrable, then $J$ may only be tamed by $\omega$. 
A straightforward calculation gives the following generalisation of [19, Proposition 5]:

Proposition 2.1. The map

$$
\begin{aligned}
\Phi:(\mathbb{R} \times \mathbb{R} \times V, J) & \longrightarrow\left(\mathbb{C} \times V, \mathrm{i} \oplus J_{V}\right) \\
(a, b, \mathbf{z}) & \longmapsto(a-\psi(\mathbf{z})+\mathrm{i} b, \mathbf{z})
\end{aligned}
$$

is a biholomorphism.

Given a holomorphic disc $\mathbb{D} \ni z \mapsto h(z) \in V$, we want to lift this to a holomorphic disc

$$
\mathbb{D} \ni z \longmapsto(a(z), b(z), h(z))
$$

in the symplectisation, with boundary in the zero level of the symplectisation, i.e. $\left.a\right|_{\partial \mathbb{D}} \equiv 0$. By Proposition 2.1, the functions $a$ and $b$ are found as follows. Let $a: \mathbb{D} \rightarrow \mathbb{R}$ be the unique solution, smooth up to the boundary, of the Poisson problem

$$
\left\{\begin{aligned}
\Delta a & =\Delta(\psi \circ h) & & \text { on } \operatorname{Int}(\mathbb{D}) \\
a & =0 & & \text { on } \partial \mathbb{D} .
\end{aligned}\right.
$$

Then $a-\psi \circ h$ is harmonic, and we may choose the function $b$ (unique up to adding a constant) such that $a-\psi \circ h+\mathrm{i} b$ is holomorphic. Notice that the function $a$ is subharmonic.

\subsection{Examples}

(1) Our first example shows how to derive the set-up of [5] in this general context. We take $V=\mathbb{C}$ with plurisubharmonic potential $\psi(x+\mathrm{i} y)=x^{2} / 2$. This yields the contact form $\mathrm{d} b+x \mathrm{~d} y$ on $\mathbb{R} \times \mathbb{C}$. Start with the holomorphic disc $h: \mathbb{D} \rightarrow \mathbb{C}$ given by inclusion. The solution $a$ of the corresponding Poisson problem - this is equation (52) in [5] - is given by $a(x, y)=\left(x^{2}+y^{2}-1\right) / 4$. For $b$ one obtains $b(x, y)=b_{0}-x y / 2$. Notice that $a-\psi \circ h+\mathrm{i} b$ is the holomorphic function $z \mapsto-\left(z^{2}+1\right) / 4+\mathrm{i} b_{0}$.

(2) For our second example we take $V=\mathbb{C}$ with plurisubharmonic potential $\psi(z)=|z|^{2} / 4$. This gives rise to the contact form $\mathrm{d} b+(x \mathrm{~d} y-y \mathrm{~d} x) / 2$ on $\mathbb{R} \times \mathbb{C}$. The solution $a$ of the Poisson problem is unchanged, but $b$ is now simply a constant function. The example in [19] is obtained by crossing this $V$ with a cotangent bundle $T^{*} Q$, on which one takes the plurisubharmonic potential $\|\mathbf{p}\|^{2} / 2$, with $\mathbf{p}$ denoting the fibre coordinate, corresponding to the canonical Liouville 1 -form on $T^{*} Q$. 


\subsection{The contact cylinder}

The contact form $\alpha_{\text {cyl }}$ on the contact cylinder $Z=\mathbb{R} \times D \times \mathbb{C}^{n-1}$ derives from the plurisubharmonic potential

$$
\psi\left(z_{0} ; z_{1}, \ldots, z_{n-1}\right):=\frac{1}{4}\left|z_{0}\right|^{2}+\frac{1}{2} \sum_{j=1}^{n-1} y_{j}^{2}
$$

on $D \times \mathbb{C}^{n-1}$, where $z_{j}=x_{j}+\mathrm{i} y_{j}, j=0,1, \ldots, n-1$.

Similar to Example 2.2 (2), for any choice of parameters $b \in \mathbb{R}, \mathbf{s}, \mathbf{t} \in \mathbb{R}^{n-1}$, we have the holomorphic discs

$$
\begin{aligned}
u_{\mathbf{s}, b}^{\mathbf{t}}: & \mathbb{D} \longrightarrow \begin{array}{c}
\mathbb{R} \times \mathbb{R} \times D^{2 n} \\
z
\end{array} \\
z & \longmapsto\left(\frac{1}{4}\left(|z|^{2}-1\right), b, z, \mathbf{s}+\mathbf{i t}\right),
\end{aligned}
$$

lifting the obvious holomorphic discs in $D \times \mathbb{C}^{n-1}$. The disc $u_{\mathbf{s}, b}^{\mathbf{t}}$ has boundary on the Lagrangian cylinder

$$
L^{\mathbf{t}}:=\{0\} \times \mathbb{R} \times S^{1} \times \mathbb{R}^{n-1} \times\{\mathbf{t}\}
$$

in $\mathbb{R} \times Z$. These Lagrangian cylinders foliate $\partial(\{0\} \times Z)$.

\section{The moduli space of holomorphic dises}

We now form the contact manifold $\left(\widehat{\mathbb{R}}^{2 n+1}, \hat{\alpha}\right)$ as explained in Section 1.2. Let

$$
\left(W:=\mathbb{R} \times \widehat{\mathbb{R}}^{2 n+1}, \omega:=\mathrm{d}(\tau \hat{\alpha})\right)
$$

be its symplectisation, where $\tau: \mathbb{R} \rightarrow \mathbb{R}^{+}$is a smooth function with $\tau^{\prime}>0$ and $\tau(a)=\mathrm{e}^{a}$ for $a \geq 0$. The freedom of choosing $\tau$ on $\{a<0\}$ is required for the asymptotic analysis cited in Section 4.

\subsection{The almost complex structure}

Choose $b_{0}, r, R \in \mathbb{R}^{+}$with $r<1$ such that $\varphi(\partial M)$ is contained in the interior of the box

$$
B:=\left[-b_{0}, b_{0}\right] \times D_{r}^{2} \times D_{R}^{2 n-2} \subset Z,
$$

where $D_{\rho}^{2 k} \subset \mathbb{C}^{k}$ denotes a closed $2 k$-disc of radius $\rho$. We write $\widehat{B}$ for the result of gluing $M$ into this box, in other words,

$$
\widehat{\mathbb{R}}^{2 n+1}=\widehat{B} \cup_{\partial B}\left(\left(\mathbb{R} \times \mathbb{C} \times \mathbb{C}^{n-1}\right) \backslash \operatorname{Int}(B)\right) .
$$


We shall also have occasion to use the notation $\widehat{Z}$ for the cylinder $Z$ with $M$ glued in, that is,

$$
\widehat{Z}=\widehat{\mathbb{R}}^{2 n+1} \backslash\left(\mathbb{R} \times(\mathbb{C} \backslash \operatorname{Int}(D)) \times \mathbb{C}^{n-1}\right) .
$$

On the symplectic manifold $(W, \omega)$ we choose an almost complex structure $J$ compatible with $\omega$ subject to the following conditions:

(J1) On the complement of $\mathbb{R} \times \operatorname{Int}(\widehat{B})$, the almost complex structure $J$ equals the one described in Section 2.1.

(J2) On $\mathbb{R} \times \operatorname{Int}(\widehat{B})$, we make a generic choice (in a sense explained in Section 5.2) of an $\mathbb{R}$-invariant almost complex structure $J$ preserving ker $\hat{\alpha}$ and satisfying $J\left(\partial_{a}\right)=R_{\hat{\alpha}}$.

Condition (J1) will allow us to prove that holomorphic discs in the relevant region are standard. Condition (J2) implies that the breaking of holomorphic discs corresponds to cylindrical ends asymptotic to Reeb orbits.

\subsection{The moduli space}

We now consider holomorphic discs (smooth up to the boundary) of the form

$$
u=(a, f):(\mathbb{D}, \partial \mathbb{D}) \longrightarrow\left(W=\mathbb{R} \times \widehat{\mathbb{R}}^{2 n+1}, L^{\mathbf{t}}\right)
$$

i.e. with Lagrangian boundary condition, where $\mathbf{t}$ is allowed to vary over $\mathbb{R}^{n-1}$. We shall call the value of $\mathbf{t}$ corresponding to a given $u$ the 'boundary level' of the holomorphic disc.

We define $\mathcal{W}$ to be the moduli space of such discs $u$, which are supposed to satisfy the following conditions:

(M1) The relative homology class $[u] \in H_{2}\left(W, L^{\mathbf{t}}\right)$, with $\mathbf{t}$ equal to the boundary level of $u$, equals that of $u_{\mathbf{s}, b}^{\mathbf{t}}$ for some $b \in \mathbb{R}, \mathbf{s} \in \mathbb{R}^{n-1}$, where $|b|,|\mathbf{s}|$ are large (such that $u_{\mathbf{s}, b}^{\mathbf{t}}$ may be regarded as a holomorphic disc in $W$ ).

(M2) For $k=0,1,2$ we have $u\left(\mathrm{i}^{k}\right) \in L^{\mathbf{t}} \cap\left\{z_{0}=\mathrm{i}^{k}\right\}$.

Let $u=(a, f)$ be a holomorphic disc satisfying (M1). By the maximum principle, $f(\mathbb{D})$ is contained in $\widehat{Z}$, see Lemma 3.6. By the boundary lemma of E. Hopf, applied to a small disc in $\mathbb{D}$ touching a given boundary point and mapping to the complement of $\mathbb{R} \times \operatorname{Int}(\widehat{B})$, so that the $z_{0}$-component of $u$ is defined and holomorphic on that small disc, the boundary $u(\partial \mathbb{D})$ is transverse to

$$
\{0\} \times \mathbb{R} \times\left\{\mathrm{e}^{\mathrm{i} \theta}\right\} \times \mathbb{R}^{n-1} \times\{\mathbf{t}\} \subset L^{\mathbf{t}}
$$

for each $\mathrm{e}^{\mathrm{i} \theta} \in S^{1}$ and, by (M1), in fact positively transverse. Thus, condition (M2) fixes a parametrisation of $u$. 


\subsection{Properties of the holomorphic dises}

Here we collect some basic properties of the $\operatorname{discs} u \in \mathcal{W}$.

Lemma 3.1. The Maslov index $\mu$ of any disc $u \in \mathcal{W}$, i.e. the index of the bundle pair $\left(u^{*} T W,\left(\left.u\right|_{\partial \mathbb{D}}\right)^{*} T L^{\mathbf{t}}\right)$, equals 2 .

Proof. We appeal to the axiomatic definition of the Maslov index in [17, Section C.3]. For the disc $u_{0}:=u_{\mathbf{0}, 0}^{\mathbf{t}}$ in $\mathbb{R} \times \mathbb{R}^{2 n+1}$, the bundle $u_{0}^{*} T\left(\mathbb{R} \times \mathbb{R}^{2 n+1}\right)$ is a trivial $\mathbb{C}^{n+1}$-bundle. The fibre of the totally real subbundle $\left(\left.u_{0}\right|_{\partial \mathbb{D}}\right)^{*} T L^{\mathbf{t}}$ over $\mathrm{e}^{\mathrm{i} \theta} \in \partial \mathbb{D}$ is given by $\mathbb{R i} \oplus \mathbb{R} \mathrm{ie}^{\mathrm{i} \theta} \oplus \mathbb{R}^{n-1}$. So the normalisation property of the Maslov index implies $\mu\left(u_{\mathbf{0}, 0}^{\mathbf{t}}\right)=2$.

By the homotopy invariance of the Maslov index, we have $\mu\left(u_{\mathbf{s}, b}^{\mathbf{t}}\right)=2$ for all standard discs $u_{\mathbf{s}, b}^{\mathbf{t}}$ in $W$. Finally, given any $u \in \mathcal{W}$, we may choose $u_{\mathbf{s}, b}^{\mathbf{t}}$ in the same relative homology class, so that $u-u_{\mathbf{s}, b}^{\mathbf{t}}$ is a boundary. This implies $\mu(u)=2$.

Lemma 3.2. Each disc $u \in \mathcal{W}$ has symplectic energy $\int_{\mathbb{D}} u^{*} \omega$ equal to $\pi$.

Proof. Choose a standard disc $u_{\mathbf{s}, b}^{\mathbf{t}}$ in the same relative class in $H_{2}\left(W, L^{\mathbf{t}}\right)$ as $u$. Then in particular $[\partial u]=\left[\partial u_{\mathbf{s}, b}^{\mathbf{t}}\right]$ in $H_{1}\left(L^{\mathbf{t}}\right)$. Since $L^{\mathbf{t}}$ is Lagrangian, the pull-back of the 1-form $\hat{\alpha}$ to $L^{\mathbf{t}}$ is closed, and hence

$$
\int_{\partial u} \hat{\alpha}=\int_{\partial u_{\mathrm{s}, b}^{\mathrm{t}}} \hat{\alpha}
$$

One then computes

$$
\int_{u} \omega=\int_{\partial u} \hat{\alpha}=\int_{\partial u_{\mathrm{s}, b}^{\mathrm{t}}} \hat{\alpha}=\int_{\partial u_{\mathrm{s}, b}^{\mathrm{t}}} \alpha_{\mathrm{cyl}}=\pi
$$

Remark 3.3. By the same argument we see that any non-constant holomorphic disc in $W$ with boundary on $L^{\mathbf{t}}$ has symplectic energy in $\pi \mathbb{N}$.

Recall that a holomorphic disc $u$ is called simple if there are no two disjoint non-empty open subsets $U, V \subset \mathbb{D}$ such that $u(U)=u(V)$. The next two lemmata will be used to establish transversality for our moduli space.

Lemma 3.4. All discs $u \in \mathcal{W}$ are simple.

Proof. According to [15, Theorem A], the homology class $[u] \in H_{2}\left(W, L^{\mathbf{t}}\right)$ of a holomorphic disc with totally real boundary condition can be decomposed into positive multiples of homology classes represented by simple discs, which are obtained from a decomposition of $\mathbb{D}$. Since the class $[u]=\left[u_{\mathbf{s}, b}^{\mathbf{t}}\right] \in H_{2}\left(W, L^{\mathbf{t}}\right)$ is indecomposable by Lemma 3.2 and Remark 3.3, the disc $u$ itself must be simple. 
Simplicity of the discs $u=(a, f)$ will not be quite enough for our purposes. We shall also need simplicity of $f$ in the sense of the following lemma, $c f$. [13, Theorem 1.14]. Here $\pi$ denotes the projection of $T M$ onto ker $\hat{\alpha}$ along the Reeb vector field $R_{\hat{\alpha}}$.

Lemma 3.5. For each $u=(a, f) \in \mathcal{W}$, the set

$$
F_{\text {inj }}:=\left\{z \in \mathbb{D}: \pi \circ T_{z} f \neq 0, f^{-1}(f(z))=\{z\}\right\}
$$

of ' $f$-injective points' is open and dense in $\mathbb{D}$.

Proof. The combination of defining conditions for $F_{\text {inj }}$ is open, so we need only show that $F_{\text {inj }}$ is dense in $\mathbb{D}$. We begin with three observations about the behaviour of the holomorphic discs $u$.

First of all, in a neighbourhood of the boundary $\partial \mathbb{D} \subset \mathbb{D}$ we can write $f$ in components as $f=(b, \mathbf{h})=\left(b, h_{0}, \ldots, h_{n-1}\right)$ with each $h_{j}$ holomorphic. By the comment in Section 3.2, $\left.h_{0}\right|_{\partial \mathbb{D}}$ is an immersion, hence $\left.\pi \circ T f\right|_{\partial \mathbb{D}} \neq 0$. Moreover, a variant of the Carleman similarity principle [5, pages 1315/6] implies that the set $\left\{z \in \mathbb{D}: \pi \circ T_{z} f=0\right\}$ is finite.

Secondly, the boundary $\partial \mathbb{D}$ maps under $f$ to $\mathbb{R} \times S^{1} \times \mathbb{C}^{n-1}$. Near any point in $\operatorname{Int}(\mathbb{D})$ that putatively maps to $\mathbb{R} \times(\mathbb{C} \backslash \operatorname{Int}(D)) \times \mathbb{C}^{n-1}$, we could write $f=(b, \mathbf{h})$ as above, and we would find that $h_{0}$ violates the maximum principle. We conclude in particular that there are no mixed intersections of the holomorphic disc $u$, i.e. pairs of an interior and a boundary point with the same image.

Thirdly, from the work in [22] it follows that the immersion $\left.u\right|_{\partial \mathbb{D}}=\left(0,\left.f\right|_{\partial \mathbb{D}}\right)$ has at most finitely many double points. Otherwise the respective preimages would accumulate in two separate points - for in a common limit point the differential $T u$ would be singular - and [22, Lemma 4.2] would imply that the differentials $T u$ in the two limit points are collinear over $\mathbb{R}$. Furthermore, by Lemma 3.6 (i) below, the collinearity factor would have to be positive. Then [22, Lemma 4.3] would imply that $u$ is not simple, contradicting the preceding lemma.

From these last two observations we infer that $F_{\text {inj }}$ contains $\partial \mathbb{D}$ with the exception of at most finitely many points, and in particular is non-empty.

Now we prove that $F_{\text {inj }}$ is dense, arguing by contradiction. If $F_{\text {inj }}$ were not dense, the set $\operatorname{Int}(\mathbb{D}) \backslash F_{\text {inj }}$ would have non-empty interior. By the preceding observations we can find an open subset $U \subset \operatorname{Int}(\mathbb{D})$ such that for each $z \in U$ the set $f^{-1}(f(z)) \subset \operatorname{Int}(\mathbb{D})$ contains more than just the point $z$, and such that $\pi \circ T_{w} f \neq 0$ in all points $w \in f^{-1}(f(U))$. The latter implies that the points in $f^{-1}(f(z))$ are isolated, and hence finite in number.

What follows is an explication of an argument in [13, page 459]. Fix a point $z_{0} \in U$ and write $f^{-1}\left(f\left(z_{0}\right)\right)=\left\{z_{0}, z_{1}, \ldots, z_{N}\right\}$. Choose pairwise disjoint (and disjoint from $U$ ) open neighbourhoods $U_{k} \subset \operatorname{Int}(\mathbb{D})$ of $z_{k}, k=1, \ldots, N$, such that $\left.f\right|_{U_{k}}$ is an embedding. By a compactness argument, $U$ can be chosen so small that

$$
f(U) \subset \bigcup_{k=1}^{N} f\left(U_{k}\right),
$$


and such that $\left.f\right|_{U}$ is likewise an embedding. Choose relatively compact neighbourhoods $U_{k}^{\prime} \subset U_{k}$ of $z_{k}, k=1, \ldots, N$. By shrinking $U$ to a smaller neighbourhood of $z_{0}$, we can ensure that

$$
f(U) \subset \bigcup_{k=1}^{N} f\left(U_{k}^{\prime}\right) .
$$

Set $A_{k}:=\left(\left.f\right|_{U}\right)^{-1}\left(f\left(U_{k}^{\prime}\right)\right) \subset U$. If $A_{1}$ has non-empty interior, we can shrink $U$ such that $f(U) \subset f\left(U_{1}^{\prime}\right)$ (but $U$ need no longer be a neighbourhood of $z_{0}$ ). The argument then concludes as in [13, pages 459/60], leading to a contradiction to $u$ being simple. If $A_{1}$ has empty interior, so that $U \backslash A_{1}$ is dense in $U$, we find that

$$
f(U) \subset \bigcup_{k=2}^{N} f\left(\overline{U_{k}^{\prime}}\right) \subset \bigcup_{k=2}^{N} f\left(U_{k}\right) .
$$

The argument concludes inductively.

\subsection{Bounds on the holomorphic discs}

In the next lemma we collect some restrictions on the image $u(\mathbb{D})$ of the holomorphic discs $u \in \mathcal{W}$.

Lemma 3.6. For $u=(a, f) \in \mathcal{W}$ we have:

(i) $a<0$ on $\operatorname{Int}(\mathbb{D})$.

(ii) $f(\operatorname{Int}(\mathbb{D}))$ is contained in the interior of $\widehat{Z}$, i.e.

$$
f(\operatorname{Int}(\mathbb{D})) \cap\left(\mathbb{R} \times(\mathbb{C} \backslash \operatorname{Int}(D)) \times \mathbb{C}^{n-1}\right)=\emptyset .
$$

Proof. (i) The holomorphicity of $u=(a, f)$ (with respect to an almost complex structure preserving ker $\hat{\alpha}$ and satisfying $\left.J\left(\partial_{a}\right)=R_{\hat{\alpha}}\right)$ implies $f^{*} \hat{\alpha}=-\mathrm{d} a \circ \mathrm{i}$, so $a$ is subharmonic. We have $\left.a\right|_{\partial \mathbb{D}} \equiv 0$, but $a$ cannot be identically zero on all of $\mathbb{D}$, for otherwise we would have $f^{*} \alpha \equiv 0$ and $f^{*} \mathrm{~d} \alpha \equiv 0$, which would imply that $u$ has zero symplectic energy density and hence is constant, contradicting (M1). The strong maximum principle for $a$ then implies the claim.

(ii) Near the points of $\mathbb{D}$ mapping under $f$ to $\mathbb{R} \times(\mathbb{C} \backslash \operatorname{Int}(D)) \times \mathbb{C}^{n-1}$ we can write this map in components as $f=(b, \mathbf{h})$. If $f(\operatorname{Int}(\mathbb{D}))$ were not contained in $\operatorname{Int}(Z)$, we would find that the map $h_{0}$ is defined and locally constant on a nonempty open and closed subset of $\mathbb{D}$, and hence on all of $\mathbb{D}$, contradicting the homological assumption (M1).

Since a generic choice of the almost complex structure $J$ is only allowed on $\mathbb{R} \times \operatorname{Int}(\widehat{B})$, this can be used to guarantee regularity in the sense of $[17$, Definition 3.1.4] only for those holomorphic discs that pass through this "perturbation domain', see [17, Remark 3.2.3]. We therefore want to show that all other discs belong to the standard family $u_{\mathbf{s}, b}^{\mathbf{t}}$, where transversality is obvious. This will be used below to show that $\mathcal{W}$ is actually a manifold. 
Lemma 3.7. Let $u=(a, f) \in \mathcal{W}$. If $f(\mathbb{D}) \subset \widehat{\mathbb{R}}^{2 n+1} \backslash \operatorname{Int}(\widehat{B})$, then $u=u_{\mathbf{s}, b}^{\mathbf{t}}$ for some $\mathbf{s} \in \mathbb{R}^{n-1}, b \in \mathbb{R}$, and $\mathbf{t}$ equal to the boundary level of $u$.

Proof. Since $f$ maps to the complement of $\operatorname{Int}(\widehat{B})$, we can write it globally as $f=$ $(b, \mathbf{h})$, with every component $h_{j}$ of $\mathbf{h}$ a holomorphic map $\mathbb{D} \rightarrow \mathbb{C}$. The boundary condition for $u$ means that for $j=1, \ldots, n-1$ we have $\operatorname{Im} h_{j}=t_{j}$ on $\partial \mathbb{D}$. The minimum and maximum principle for harmonic functions implies that $\operatorname{Im} h_{j}=t_{j}$ on all of $\mathbb{D}$. Hence, by the open mapping theorem, $\operatorname{Re} h_{j}=: s_{j}$ is likewise constant on $\mathbb{D}$ for $j=1, \ldots, n-1$.

The component $h_{0}$ is a holomorphic disc in $\mathbb{C}$ with $\left.h_{0}\right|_{\partial \mathbb{D}}$ an orientationpreserving diffeomorphism of $\partial \mathbb{D}, c f$. the comment after condition (M2). The argument principle implies that $h_{0}$ is an orientation-preserving automorphism of $\mathbb{D}$, and then (M2) forces $h_{0}=\mathrm{id}_{\mathbb{D}}$.

By Proposition 2.1, the function

$$
z \longmapsto a(z)-\frac{1}{4}\left|h_{0}(z)\right|^{2}-\frac{1}{2} \sum_{j=1}^{n-1}\left(\operatorname{Im} h_{j}\right)^{2}=a(z)-\frac{1}{4}|z|^{2}-\frac{1}{2} \sum_{j=1}^{n-1}\left(\operatorname{Im} h_{j}\right)^{2}
$$

on $\mathbb{D}$ is harmonic, taking the constant value $-1 / 4-|\mathbf{t}|^{2} / 2$ on $\partial \mathbb{D}$, hence it is constant on $\mathbb{D}$. This means that the imaginary part $b$ that makes this into a holomorphic function must also be constant. Solving for $a(z)$ we get

$$
a(z)=\frac{1}{4}\left(|z|^{2}-1\right) \text { on } \mathbb{D},
$$

i.e. $u=u_{\mathbf{s}, b}^{\mathbf{t}}$.

The next lemma will allow us to control the degree of the evaluation map ev. It says that non-standard discs can never reach $b$-levels with $|b|>b_{0}$. This is also relevant for compactness.

Lemma 3.8. Let $u=(a, f) \in \mathcal{W}$. On the closed set $A:=f^{-1}\left(\widehat{\mathbb{R}}^{2 n+1} \backslash \operatorname{Int}(\widehat{B})\right) \subset$ $\mathbb{D}$, which includes the whole boundary $\partial \mathbb{D}$ in its interior, we write $f=(b, \mathbf{h})$. If the function $b$ takes values outside $\left[-b_{0}, b_{0}\right]$, then $f$ maps to a b-level set $\left\{b_{1}\right\} \times D \times$ $\mathbb{C}^{n-1}$ with $\left|b_{1}\right|>b_{0}$ and hence, by the preceding lemma, the holomorphic curve $u$ equals $u_{\mathbf{s}, b_{1}}^{\mathbf{t}}$ for some $\mathbf{s}$ and $\mathbf{t}$.

Proof. Choose $z_{*} \in A$ with $b_{*}:=b\left(z_{*}\right)$ of maximal absolute value. Notice that $z_{*}$ is an interior point of $A$. By Proposition 2.1, the function

$$
g:=a-\frac{1}{4}\left|h_{0}\right|^{2}-\frac{1}{2} \sum_{j=1}^{n-1}\left(\operatorname{Im} h_{j}\right)^{2}+\mathrm{i} b
$$

is holomorphic on $\operatorname{Int}(A)$. We should now like to argue with the maximum principle that the imaginary part $b$ of $g$ has to be constant equal to $b_{*}$ on an open and closed 
subset of $\mathbb{D}$. If $z_{*} \in \operatorname{Int}(\mathbb{D})$, this inference is indeed conclusive, just as in part (ii) of Lemma 3.6. If $z_{*} \in \partial \mathbb{D}$, we reason as follows.

The real part of the holomorphic function $g$ takes the constant value $a_{\partial}:=$ $-1 / 4-|\mathbf{t}|^{2} / 2$ on $\partial \mathbb{D} \subset \operatorname{Int}(A)$. It follows that the function can be extended by Schwarz reflection to the complementary set $\bar{A}$ of $A$ in $\hat{\mathbb{C}} \backslash \mathbb{D}$, with $\hat{\mathbb{C}}$ denoting the Riemann sphere. Indeed, the holomorphic function $\mathrm{i}\left(g-a_{\partial}\right)$ takes real values on $\partial \mathbb{D}$, so the Schwarz reflection principle applies to this function, and we simply transform the extension via the map $w \mapsto-\mathrm{i} w+a_{\partial}$ to a holomorphic extension of $g$. Now $z_{*}$ is an interior point of $A \cup \bar{A}$, and we conclude as before with the maximum principle.

Finally, we establish a $C^{0}$-bound in the $\mathbb{C}^{n-1}$-direction on non-standard discs.

Lemma 3.9. Let $u=(a, f) \in \mathcal{W}$. If $f(\mathbb{D})$ intersects

$$
\mathbb{R} \times \mathbb{C} \times\left(\mathbb{C}^{n-1} \backslash D_{R+\sqrt{2}}^{2 n-2}\right),
$$

then $u$ equals one of the standard discs $u_{\mathbf{s}, b}^{\mathbf{t}}$.

Proof. Consider the open subset

$$
G:=f^{-1}\left(\mathbb{R} \times \mathbb{C} \times\left(\mathbb{C}^{n-1} \backslash D_{R}^{2 n-2}\right)\right) \subset \mathbb{D},
$$

which will be non-empty under the assumption on $f$ in the lemma. On the closure $\bar{G}$ of $G$ we write $f=(b, \mathbf{h})$ as before. For convenience we set $\mathbf{k}:=\left(h_{1}, \ldots, h_{n-1}\right)$. Consider the subharmonic function $k:=\left|h_{1}\right|^{2}+\cdots+\left|h_{n-1}\right|^{2}=|\mathbf{k}|^{2}$.

Write $\partial G$ for the topological boundary of $G$ in $\mathbb{D}$. We have $\left.k\right|_{\partial G} \equiv R^{2}$, so the maximum of $k$ on $\bar{G}$ must be attained at a point in $G \cap \partial \mathbb{D}$ (in particular, this intersection must be non-empty).

If $\bar{G}=\mathbb{D}$, we are done by Lemma 3.7. Otherwise, we perform Schwarz reflection on the holomorphic function $h_{j}-\mathrm{i} t_{j}$, which is possible since $\operatorname{Im} h_{j} \equiv t_{j}$ on $\partial \mathbb{D}$. To the extended function we add it $t_{j}$ again to obtain the extension of $h_{j}$. Geometrically, this corresponds to a reflection of $h_{j}(G)$ in the line $\left\{z_{j}=\mathrm{i} t_{j}\right\} \subset \mathbb{C}$.

Write $S$ for the compact subset of the Riemann sphere given as the union of $\bar{G}$ and its reflected copy, and continue to write $k$ for the extension of the plurisubharmonic function to $S$. Beware that $k$ may take larger values on $S$ than on $\bar{G}$.

Choose a point $s_{0} \in G \cap \partial \mathbb{D}$ where $\left.k\right|_{\bar{G}}$ attains its maximum $(R+\delta)^{2}$. Now consider an open $\delta$-ball $B_{\delta}$ about the point $\mathbf{k}\left(s_{0}\right) \in \mathbb{C}^{n-1}$. Then $\mathbf{k}(\partial G)$ is contained in the complement of $B_{\delta}$, and since the extension of $\mathbf{k}$ to $S$ was obtained by Schwarz reflection along $\partial \mathbb{D} \ni s_{0}$, the full boundary $\mathbf{k}(\partial S)$ after reflection will likewise be contained in the complement of $B_{\delta}$.

This allows us to apply the monotonicity lemma [14, Theorem II.1.3], which tells us that the area of $\mathbf{k}(S) \cap \bar{B}_{\delta}$ is bounded from below by $\pi \delta^{2}$. (In [14] the estimate is given in the form const. $\delta^{2}$; in the present Euclidean setting the constant 
$\pi$ comes from the classical isoperimetric inequality.) So the area of $\mathbf{k}(\bar{G}) \cap \bar{B}_{\delta}$ is bounded from below by $\pi \delta^{2} / 2$, and from above by the energy $\pi$ of $u$. This implies $\delta \leq \sqrt{2}$.

To sum up: Any holomorphic disc $u$ whose k-component stays outside $D_{R}^{2 n-2}$ is standard; for all other discs the k-component stays inside $D_{R+\sqrt{2}}^{2 n-2}$.

\section{Compactness}

In this section we establish, under the assumption $\inf _{0}(\alpha)>\pi$, compactness of the truncated moduli space

$$
\mathcal{W}^{\prime}:=\left\{u=(a, f) \in \mathcal{W}: f(\mathbb{D}) \subset\left[-b_{0}, b_{0}\right] \times D \times D_{R+\sqrt{2}}^{2 n-2}\right\},
$$

i.e. the space obtained from $\mathcal{W}$ by cutting off ends containing standard discs only.

\subsection{Variable boundary condition}

The holomorphic discs $u \in \mathcal{W}$ have boundary on the Lagrangian cylinder $L^{\mathbf{t}}$, which varies with the parameter $\mathbf{t} \in \mathbb{R}^{n-1}$. It is possible to fix the boundary condition, at the cost of allowing the almost complex structure to vary. This is done with the help of a flow that enables us to identify different copies of $L^{\mathbf{t}}$. That flow will also provide explicit charts when we discuss transversality.

Start with a constant vector field $\mathbf{v}$ on the space $\operatorname{Im} \mathbb{C}^{n-1}$ of $\mathbf{t}$-coordinates, and regard this as a vector field on $\mathbb{R} \times \mathbb{R} \times \mathbb{C} \times \mathbb{C}^{n-1}$. Cut this off with a bump function supported near

$$
\{0\} \times\left[-b_{0}, b_{0}\right] \times S^{1} \times \mathbb{C}^{n-1}
$$

and identically 1 in a smaller neighbourhood of that set. Then write $\psi_{t}^{\mathbf{v}}$ for the flow of this vector field.

For a sequence $u_{v}$ of holomorphic discs of level $\mathbf{t}_{v} \rightarrow \mathbf{t}_{0}$, we can then use the maps $\psi_{1}^{\mathbf{t}_{v}-\mathbf{t}_{0}}$ to pull back the $u_{v}$ to $J_{v}$-holomorphic discs of level $\mathbf{t}_{0}$, where $J_{v}:=$ $\left(\psi_{1}^{\mathbf{t}_{v}-\mathbf{t}_{0}}\right)^{*} J$ is $C^{\infty}$-convergent to $J$ and coincides with $J$ outside the neighbourhood described in the preceding paragraph.

\subsection{Proof of compactness}

Now we apply this construction to the truncated moduli space $\mathcal{W}^{\prime}$. Consider a sequence $\left(u_{v}\right)$ of holomorphic discs $u_{v}=\left(a_{v}, f_{v}\right) \in \mathcal{W}^{\prime}$. Then, in particular the levels $\mathbf{t}_{v}$ will be contained in the compact set $D_{R+\sqrt{2}}^{n-1}$. Hence, after passing to a subsequence, we may assume that $\mathbf{t}_{v} \rightarrow \mathbf{t}_{0}$ for some $\mathbf{t}_{0} \in D_{R+\sqrt{2}}^{n-1}$. With the construction from the preceding section we may take the $u_{v}$ to be $J_{v}$-holomorphic discs of fixed boundary level $\mathbf{t}_{0}$. The almost complex structures $J_{v}$ equal $J$ outside 
a neighbourhood of $\{0\} \times\left[-b_{0}, b_{0}\right] \times S^{1} \times \mathbb{C}^{n-1}$ and converge to $J$ in the $C^{\infty}$ topology. By Lemma 3.2, all discs $u_{\nu}$ have symplectic energy equal to $\pi$.

We claim that there is a uniform bound on $\max _{\mathbb{D}}\left|\nabla u_{\nu}\right|$. Here $|$.$| denotes the$ norm corresponding to an $\mathbb{R}$-invariant metric on $W$ of the form $\mathrm{d} a^{2}+g_{\widehat{\mathbb{R}}^{2 n+1}}$, with $g_{\widehat{\mathbb{R}}^{2 n+1}}$ any Riemannian metric on $\widehat{\mathbb{R}}^{2 n+1}$. The mean value theorem then gives a uniform $C^{0}$-bound on $\left(a_{v}\right)$, and compactness follows as in [11] with [17, Theorem B.4.2].

Bubbling off analysis as in [11, Section 6] shows that, a priori, the following phenomena might occur:

- bubbling of spheres

- bubbling of finite energy planes

- breaking

- bubbling of discs (this can only happen at boundary points).

The first is impossible in an exact symplectic manifold. The second and third phenomenon are precluded by the assumption $\inf _{0}(\alpha)>\pi$ and the energy estimate from Lemma 3.2, $c f$. [11, page 548], since a finite energy plane in a symplectisation is asymptotic to a contractible Reeb orbit. Notice that this rules out any kind of bubbling at interior points.

This leaves the bubbling of discs at boundary points. By Remark 3.3, there could be at best a single bubble disc at the boundary, taking away the full energy $\pi$, $c f$. [17, Theorem 4.6.1]. But the $C_{\text {loc }}^{\infty}$ convergence on the complement of the bubble point, together with condition (M2), is incompatible with a ghost disc.

\section{Transversality}

The purpose of this section is to show that the truncated moduli space $\mathcal{W}^{\prime}$ is a smooth, oriented manifold with boundary. As usual, this is achieved by proving transversality results in the setting of $W^{1, p}$-maps for some $p>2$. Smoothness of the holomorphic discs is then implied by elliptic regularity.

Let $\mathcal{B}$ denote the space of $W^{1, p}$-maps

$$
u:(\mathbb{D}, \partial \mathbb{D}) \longrightarrow\left(W,\{0\} \times \widehat{\mathbb{R}}^{2 n+1}\right),
$$

where $u(\partial \mathbb{D})$ is supposed to be contained in $L^{\mathbf{t}}$ for some $\mathbf{t} \in \mathbb{R}^{n-1}$, and $u$ is required to satisfy the homological condition (M1) from Section 3.2. Write $\mathcal{B}^{\mathbf{t}} \subset \mathcal{B}$ for the subspace of discs corresponding to a fixed boundary level $\mathbf{t}$.

The space $\mathcal{B}^{\mathrm{t}}$ is a (separable) Banach manifold modelled on the Banach space of $W^{1, p}$-sections of $u^{*}\left(T W, T L^{\mathbf{t}}\right)$ (i.e. vector fields along $u$ that are tangent to $L^{\mathbf{t}}$ along the boundary); charts are obtained from such vector fields along $u$ by choosing a metric for which the submanifold $L^{\mathbf{t}}$ is totally geodesic and then applying the exponential map, see [6]. The construction from Section 4.1 shows that the map 
sending a disc $u \in \mathcal{B}$ to its level $\mathbf{t}$ gives $\mathcal{B}$ the structure of a locally trivial fibration over $\mathbb{R}^{n-1}$ with fibre $\mathcal{B}^{t}$. Tangent vectors at $u \in \mathcal{B}$ can be written uniquely as $\mathfrak{u}+\left.\mathfrak{v}\right|_{u}$, where $\mathfrak{u} \in T_{u} \mathcal{B}^{\mathbf{t}}$, and $\mathfrak{v}$ is a vector field as in Subsection 4.1 coming from a constant vector field $\overline{\mathfrak{v}}$ on $\operatorname{Im} \mathbb{C}^{n-1}$.

\subsection{The linearised Cauchy-Riemann operator}

Over $\mathcal{B}$ we have a Banach space bundle $\mathcal{E}$ whose fibre over the point $u \in \mathcal{B}$ is the space $L^{p}\left(u^{*} T W\right)$ of $L^{p}$-vector fields along $u$; see for instance [1, Proposition 6.13] for the construction of the bundle structure. This bundle inherits the local product structure from $\mathcal{B}$.

Fix an almost complex structure $J$ on $W$ satisfying the conditions (J1) and (J2). The Cauchy-Riemann operator $u \mapsto u_{x}+J(u) u_{y}$ defines a section of $\mathcal{E}$. In order to discuss transversality, we need to compute the vertical differential $D_{u}$ of this section at $u \in \mathcal{B}$. To this end, consider a path of holomorphic curves

$$
u^{s}:=\psi_{1}^{s \mathfrak{v}} \circ \exp _{u}(s \mathfrak{u})
$$

for $s$ in some small interval around 0 , where $\psi$ denotes the flow as in Section 4.1. This path is tangent to $\mathfrak{u}+\left.\mathfrak{v}\right|_{u}$ in $s=0$. Let $\nabla$ be a torsion-free connection on $T W$. Write

$$
\nabla_{s}=\left.\left(\nabla_{\partial u^{s} / \partial s}\right)\right|_{s=0}, \quad \nabla_{x}=\left.\left(\nabla_{\partial u^{s} / \partial x}\right)\right|_{s=0},
$$

and likewise $\nabla_{y}$. Since the torsion of $\nabla$ vanishes, we have

$$
\nabla_{s} \frac{\partial u^{s}}{\partial x}=\nabla_{x} \frac{\partial u^{s}}{\partial s}=\nabla_{x}(\mathfrak{u}+\mathfrak{v}),
$$

and similarly for $\partial u^{s} / \partial y$. Hence

$$
\begin{aligned}
D_{u}\left(\mathfrak{u}+\left.\mathfrak{v}\right|_{u}\right) & =\nabla_{s}\left(u_{x}^{s}+J\left(u^{s}\right) u_{y}^{s}\right) \\
& =\nabla_{x}(\mathfrak{u}+\mathfrak{v})+J(u) \nabla_{y}(\mathfrak{u}+\mathfrak{v})+\left(\nabla_{\mathfrak{u}+\mathfrak{v}} J\right)(u) u_{y} \\
& =D_{u}^{\mathbf{t}} \mathfrak{u}+K_{u} \overline{\mathfrak{v}}
\end{aligned}
$$

where

$$
\begin{aligned}
& D_{u}^{\mathbf{t}} \mathfrak{u}:=\nabla_{x} \mathfrak{u}+J(u) \nabla_{y} \mathfrak{u}+\left(\nabla_{\mathfrak{u}} J\right)(u) u_{y}, \\
& K_{u} \overline{\mathfrak{v}}:=\nabla_{x} \mathfrak{v}+J(u) \nabla_{y} \mathfrak{v}+\left(\nabla_{\mathfrak{v}} J\right)(u) u_{y} .
\end{aligned}
$$

The operator $\overline{\mathfrak{v}} \mapsto K_{u} \overline{\mathfrak{v}}$ is linear of order 0 in $\overline{\mathfrak{v}}$, and hence a compact operator. The restriction of $D_{u}$ to the subspace $T_{u} \mathcal{B}^{\mathbf{t}}$ equals $D_{u}^{\mathbf{t}}$, which is a Fredholm operator of index

$$
\operatorname{index}\left(D_{u}^{\mathbf{t}}\right)=\mu+n+1=n+3
$$

by the index formula [17, Theorem C.1.10] and Lemma 3.1. The subspace $\mathfrak{V}_{u} \subset$ $T_{u} \mathcal{B}$ made up of vectors of the form $\left.\mathfrak{v}\right|_{u}$ is $(n-1)$-dimensional, and it is contained 
in the kernel of $D_{u}^{\mathrm{t}}$. Hence, by the invariance under compact perturbations of both the Fredholm property and the index, see [17, Theorem A.1.4], we have - writing (1) for the zero operator -

$$
\operatorname{index}\left(D_{u}\right)=\operatorname{index}\left(D_{u}^{\mathbf{t}}+\mathbb{O}_{\mathfrak{V}_{u}}\right)=\operatorname{index}\left(D_{u}^{\mathbf{t}}\right)+n-1=2 n+2 .
$$

\subsection{Regular almost complex structures}

Given an almost complex structure $J$ on $W$ subject to the constraints (J1) and (J2), write $\widetilde{\mathcal{W}}$ for the space of holomorphic discs $u \in \mathcal{B}$, i.e. those $u$ with $u_{x}+J(u) u_{y}=$ 0 . In other words, these are holomorphic discs satisfying condition (M1).

The almost complex structure $J$ is called regular if two conditions are satisfied:

(i) $D_{u}$ is onto for all $u \in \widetilde{\mathcal{W}}$.

(ii) The evaluation map

$$
\begin{array}{ccc}
\widetilde{\mathcal{W}} & \longrightarrow & L^{\mathbf{t}} \times L^{\mathbf{t}} \times L^{\mathbf{t}} \\
u=(a, f) & \longmapsto & (f(1), f(\mathrm{i}), f(-1))
\end{array}
$$

is transverse to $L_{1}^{\mathbf{t}} \times L_{\mathrm{i}}^{\mathbf{t}} \times L_{-1}^{\mathbf{t}}$, where $L_{\mathrm{e}^{\mathrm{i} \theta}}^{\mathbf{t}}:=L^{\mathbf{t}} \cap\left\{z_{0}=\mathrm{e}^{\mathrm{i} \theta}\right\}$.

If the first condition is satisfied, $\widetilde{\mathcal{W}}$ will be a manifold of the expected dimension $2 n+2$; if in addition (ii) holds, then $\mathcal{W}$ will be a manifold of dimension $2 n-1$.

The proof that the set of regular $J$ is non-empty, in fact of second Baire category, follows the standard line of reasoning as in the proof of Theorems 3.1.5 and 3.4.1 of [17]. Selecting such a regular $J$ is the generic choice we make in (J2). For the standard discs $u_{\mathbf{s}, b}^{\mathbf{t}}$, transversality is obvious. By Lemma 3.7, all discs that are not standard pass through the region where $J$ may be chosen generically, which is sufficient to achieve transversality by [17, Remark 3.2.3]. In contrast with the set-up in [17], we are only allowed to perturb $J$ along $\xi$, keeping it compatible with $\mathrm{d} \hat{\alpha}$. But this is exactly the situation dealt with by Bourgeois in the appendix of [4]. The proof given there carries over to our situation; the essential ingredient of Bourgeois's argument is that the set of $f$-injective points is open and dense, which is precisely our Lemma 3.5.

\subsection{Orientation}

In order to speak of the degree of the evaluation map ev on $\mathcal{W} \times \mathbb{D}$, we need to put an orientation on the moduli space $\mathcal{W}$. Given the relation between $\mathcal{W}$ and $\widetilde{\mathcal{W}}$ described in the preceding section, it suffices to orient $\widetilde{\mathcal{W}}$, and that in turn amounts to showing that the determinant line bundle det $D$ over $\widetilde{\mathcal{W}}$ is oriented, since $\operatorname{ker} D_{u}=T_{u} \widetilde{\mathcal{W}}$.

Recall that the determinant line $\operatorname{det} F$ is defined for any Fredholm operator $F$ as $\operatorname{det} F=\operatorname{det} \operatorname{ker} F \otimes(\operatorname{det} \operatorname{coker} F)^{*}$. Since $D_{u}$ is surjective for all $u \in \widetilde{\mathcal{W}}$, the determinant line bundle is simply det $\operatorname{ker} D=\bigwedge^{2 n+2} \operatorname{ker} D$. In the arguments that 
follow, however, we use deformations through not necessarily surjective Fredholm operators, so we need to work with determinant lines, in general.

As we have seen, the operator $D_{u}$ splits (by slight abuse of notation) as $D_{u}=$ $D_{u}^{\mathbf{t}}+K_{u}$. The linear interpolation of $D_{u}$ to $D_{u}^{\mathbf{t}}+\mathbb{O}_{\mathfrak{V}_{u}}$ is via Fredholm operators, since $K_{u}$ is compact. It follows that $\operatorname{det}\left(D_{u}\right)=\operatorname{det}\left(D_{u}^{\mathbf{t}}+\mathbb{O}_{\mathfrak{V}_{u}}\right)$, see [7, page 680], whence

$$
\operatorname{det}\left(D_{u}\right)=\operatorname{det} D_{u}^{\mathbf{t}} \otimes \operatorname{det} \mathfrak{V}_{u}
$$

The second factor inherits a natural orientation from the orientation of $\mathbb{R}^{n-1}$. The first factor is naturally oriented by the construction in [7, Section 8.1]. Our situation is a particularly simple one, since $T L^{\mathbf{t}}$ is a trivial bundle. This implies that any bundle pair $\left(u^{*} T W,\left(\left.u\right|_{\partial \mathbb{D}}\right)^{*} T L^{\mathbf{t}}\right)$ comes with a natural trivialisation of the boundary bundle, and this suffices for the construction of a natural orientation of the determinant line bundle.

\section{Proof of Theorem 1.2}

By Sections 3 to 5 (notably Lemma 3.8), the assumption $\inf _{0}(\alpha)>\pi$ of Theorem 1.2 implies that the evaluation map

$$
\begin{aligned}
& \mathrm{ev}: \mathcal{W} \times \mathbb{D} \longrightarrow \widehat{Z} \\
& ((a, f), z) \longmapsto f(z)
\end{aligned}
$$

is a proper map of degree 1 . By Lemmata 3.7 and 3.9, we may pretend that $\mathcal{W} \times \mathbb{D}$ and $\widehat{Z}$ are - after smoothing corners - compact, oriented manifolds with boundary, without changing the homotopy type of these spaces, and that ev is a smooth degree 1 map between these manifolds.

Homotopical and homological arguments similar to the ones that follow were used by Eliashberg-Floer-McDuff, see [16].

Proposition 6.1. The manifold $\widehat{Z}$ is simply connected.

Proof. Given a loop in $\widehat{Z}$, we homotope it to an embedded circle $C$ inside $\operatorname{Int}(\widehat{Z})$ that intersects the complement of $\widehat{B}$, in other words, such that it passes through the region where all holomorphic discs (more precisely, their $f$-components) are standard. We can make the evaluation map

$$
\begin{aligned}
& \mathcal{W} \times \mathbb{D} \longrightarrow \widehat{Z} \\
& ((a, f), z) \longmapsto f(z)
\end{aligned}
$$

transverse to $C$ by a perturbation compactly supported in $\operatorname{Int}(\widehat{B})$. The preimage of $C$ under this perturbed map will then be a single circle $C^{\prime} \subset \mathcal{W} \times \mathbb{D}$ mapping with degree 1 onto $C$. The homotopy of $C^{\prime}$ to a loop in $\mathcal{W} \times\{1\}$ induces a homotopy of $C$ to a loop in the cell $\mathbb{R} \times\{1\} \times \mathbb{C}^{n-1}$. 
Lemma 6.2. Let $\phi:(P, \partial P) \rightarrow(Q, \partial Q)$ be a degree 1 map between compact, oriented m-dimensional manifolds with boundary. Then the induced homomorphism $\phi_{*}: H_{k}(P ; \mathbb{F}) \rightarrow H_{k}(Q ; \mathbb{F})$ in singular homology with coefficients in a field $\mathbb{F}$ is surjective in each degree $k \in \mathbb{N}_{0}$.

Proof. Over a field, the Kronecker pairing between homology and cohomology is non-degenerate, so equivalently we need to show injectivity of the induced homomorphism $\phi^{*}$ in cohomology.

Given a non-zero class $\beta \in H^{k}(Q)$, Poincaré duality allows us to find a class $\gamma \in H^{m-k}(Q, \partial Q)$ such that $\beta \cup \gamma$ is the orientation generator of $H^{m}(Q, \partial Q)$. Since $\phi$ is of degree 1 , we have

$$
0 \neq \phi^{*}(\beta \cup \gamma)=\phi^{*} \beta \cup \phi^{*} \gamma,
$$

which forces $\phi^{*}$ to be injective on $H^{k}(Q)$.

Proposition 6.3. The manifold $\widehat{Z}$ has the integral homology of a point.

Proof. With the preceding lemma this follows with an argument completely analogous to the proof of Proposition 6.1.

Proof of Theorem 1.2. Since $2 n \neq 3$, the smooth Schoenflies theorem tells us that the subset of $\widehat{Z}$ bounded by $\varphi(\partial M)$ and a standard ellipsoid surrounding $\varphi(\partial M)$ is diffeomorphic to a collar of $\partial M$. Hence $M$ is a strong deformation retract of $\widehat{Z}$. So by Propositions 6.1 and 6.3 , the manifold $M$ is a simply connected homology ball with boundary diffeomorphic to $S^{2 n}$. It follows that $M$ is diffeomorphic to a ball: for $n \geq 3$ we appeal to Proposition A on page 108 of Milnor's lectures [18]; for $n=2$, to Proposition C on page 110 .

\section{References}

[1] B. Aebischer, M. Borer, M. Kälin, Ch. Leuenberger and H. M. Reimann, "Symplectic Geometry", Progr. Math., Vol. 124, Birkhäuser, Basel, 1994.

[2] J. B. VAN DEN BERG, F. PASQUOTTO and R. C. VANDERVORST, Closed characteristics on non-compact hypersurfaces in $\mathbb{R}^{2 n}$, Math. Ann. 343 (2009), 247-284.

[3] J. B. VAN DEN BERG, F. PASQuOTTO, T. O. Rot and R. C. VANDERVORst, Closed characteristics on non-compact mechanical contact manifolds, J. Symplectic Geom, to appear.

[4] F. BOURGEOIS, Contact homology and homotopy groups of the space of contact structures, Math. Res. Lett. 13 (2006), 71-85.

[5] YA. Eliashberg and H. HoFer, A Hamiltonian characterization of the three-ball, Differential Integral Equations 7 (1994), 1303-1324.

[6] H. I. ElíASSON, Geometry of manifolds of maps, J. Differential Geom. 1 (1967), 169-194.

[7] K. FuKaYA, Y.-G. OH, H. OHTA and K. OnO, "Lagrangian Intersection Floer Theory: Anomaly and Obstruction, Part II", AMS/IP Stud. Adv. Math., Vol. 46.2, American Mathematical Society, Providence, RI, 2009.

[8] H. GeIgES, “An Introduction to Contact Topology", Cambridge Stud. Adv. Math., Vol. 109, Cambridge University Press, 2008. 
[9] H. Geiges, N. RötTGEN and K. Zehmisch, Trapped Reeb orbits do not imply periodic ones, Invent. Math. 198 (2014), 211-217.

[10] H. GEIGES and K. ZEHMISCH, Symplectic cobordisms and the strong Weinstein conjecture, Math. Proc. Cambridge Philos. Soc. 153 (2012), 261-279.

[11] H. GeIGES and K. ZeHMisch, How to recognize a 4-ball when you see one, Münster J. Math. 6 (2013), 525-554; erratum: 555-556.

[12] H. HOFER, Pseudoholomorphic curves in symplectizations with applications to the Weinstein conjecture in dimension three, Invent. Math. 114 (1993), 515-563.

[13] H. HOFER, K. WYSOCKI and E. ZEHNDER, Properties of pseudoholomorphic curves in symplectizations III: Fredholm theory, In: "Topics in Nonlinear Analysis", Progr. Nonlinear Differential Equations Appl., Vol. 35, Birkhäuser, Basel (1999), 381-475.

[14] C. Hummel, "Gromov's Compactness Theorem for Pseudo-holomorphic Curves", Progr. Math., Vol. 151, Birkhäuser, Basel, 1997.

[15] L. LAZZARINI, Relative frames on J-holomorphic curves, J. Fixed Point Theory Appl. 9 (2011), 213-256.

[16] D. MCDUFF, Symplectic manifolds with contact type boundaries, Invent. Math. 103 (1991), 651-671.

[17] D. MCDuff and D. SAlamon, “ $J$-holomorphic Curves and Symplectic Topology”, Amer. Math. Soc. Colloq. Publ., Vol. 52, American Mathematical Society, Providence, RI, 2004.

[18] J. MILNOR, "Lectures on the $h$-Cobordism Theorem", Princeton University Press, Princeton, NJ, 1965.

[19] K. NIEDERKRÜGER, The plastikstufe - a generalization of the overtwisted disk to higher dimensions, Algebr. Geom. Topol. 6 (2006), 2473-2508.

[20] S. SuHR and K. Zehmisch, Linking and closed orbits, Abh. Math. Semin. Univ. Hambg., to appear.

[21] A. Weinstein, Contact surgery and symplectic handlebodies, Hokkaido Math. J. 20 (1991), 241-251.

[22] K. ZeHMisch, The annulus property of simple holomorphic discs, J. Symplectic Geom. 11 (2013), 135-161.

\author{
Mathematisches Institut \\ Universität zu Köln \\ Weyertal 86-90 \\ 50931 Köln, Germany \\ geiges@math.uni-koeln.de \\ Mathematisches Institut \\ WWU Münster \\ Einsteinstraße 62 \\ 48149 Münster, Germany \\ kai.zehmisch@uni-muenster.de
}

